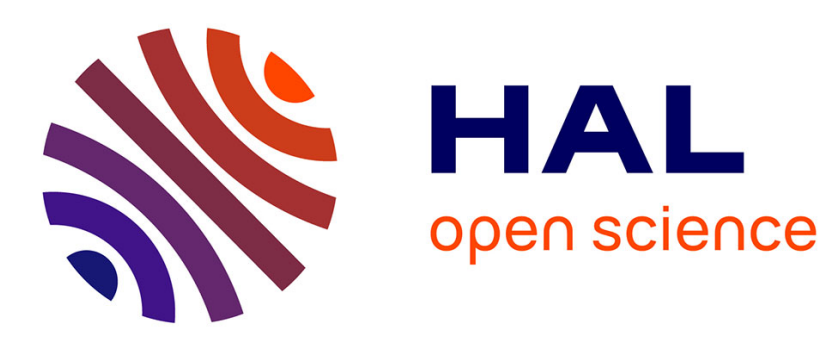

\title{
Stability of distributed delay systems via a robust approach
}

\author{
Frédéric Gouaisbaut, Yassine Ariba, Alexandre Seuret
}

\section{To cite this version:}

Frédéric Gouaisbaut, Yassine Ariba, Alexandre Seuret. Stability of distributed delay systems via a robust approach. European Control Conference (ECC), Jul 2015, Linz, Austria. pp.2068-2073. hal-01229470

\section{HAL Id: hal-01229470 \\ https://hal.science/hal-01229470}

Submitted on 16 Nov 2015

HAL is a multi-disciplinary open access archive for the deposit and dissemination of scientific research documents, whether they are published or not. The documents may come from teaching and research institutions in France or abroad, or from public or private research centers.
L'archive ouverte pluridisciplinaire HAL, est destinée au dépôt et à la diffusion de documents scientifiques de niveau recherche, publiés ou non, émanant des établissements d'enseignement et de recherche français ou étrangers, des laboratoires publics ou privés. 


\title{
Stability of distributed delay systems via a robust approach*
}

\author{
Frédéric Gouaisbaut $^{a, b}$, Yassine Ariba ${ }^{a, c}$, Alexandre Seuret ${ }^{a, d}$
}

\begin{abstract}
This paper is dedicated to the stability analysis of a class of distributed delay systems with a non constant kernel. By the use of appropriate orthogonal polynomials, this kernel is expressed as the sum of a polynomial and an additive bounded function. The resulting system is then modeled by an interconnected system between a nominal finite dimensional linear system and a infinite dimensional system. This last system is considered as a structured uncertainty and embedded into some well defined structured uncertainties. Appropriate robust control tools, i.e. quadratic separation, are then used to address the stability issue. Finally, numerical examples show the effectiveness of the proposed method.
\end{abstract}

\section{INTRODUCTION}

Since a few decades, the emergence of well established theory and potential applications led to a huge number of papers dedicated to the stability analysis of time delay systems [26]. Among them, few of them focused on the class of distributed time delay systems, for which the delay operator, via an integral action, has a cumulative effect over the past values of the dynamics. For specific integral kernels, including constant, rationals, $\gamma$ distribution function, stability of such systems can be tackled by a direct method based on pole location [3], [15], [19], [25]. For kernels which can be interpreted as impulse responses of a linear system, the system can be modeled as a linear pointwise delay system and hence classical techniques on pole location can be used (see [19]). Nevertheless, these techniques are often restricted to the case of nominal systems without uncertainties [26] and for a constant delay.

Another widely used technique for stability analysis relies on Lyapunov Theory. The construction of Lyapunov Krasovskii functionals is modified in order to take into account the distributed nature of the delay. It results some Lyapunov structure which depends on double and triple integral terms [4], [5], [11], [13], [14], [28]. Different bounding techniques are then employed to derive numerically efficient stability tests. In general, the kernel is then restricted to be a constant function over the delay interval. In order to avoid this strong constraint, another possibility, described by $\mathrm{Gu}$ et al [10], is to use a very general class of Lyapunov Krasovskii functional (see for instance [21]) and a discretization scheme to obtain numerically tractable stability conditions. This technique designed for piecewise constant delay kernel leads to a

\footnotetext{
*This work has been supported by ANR project LimICoS contract number 12 BS03 00501 .

${ }^{a}$ CNRS, LAAS, 7 avenue du Colonel Roche, 31077 Toulouse, France.

${ }^{b}$ Univ de Toulouse, UPS, LAAS, F-31400, Toulouse, France.

${ }^{c}$ Dpt of Electrical Engineering and Computer Science, ICAM, 75 avenue de Grande Bretagne, 31300 Toulouse, France

${ }^{d}$ Univ de Toulouse, LAAS, F-31400 Toulouse, France.

gouaisbaut, ariba, seuretelaas. fr
}

reduction of conservatism at the expense of the numerical burden. The case of general kernels have been studied successfully by [27] which proposed the use of new integral inequalities. These new bounding techniques which were especially designed for distributed delay systems led to new Lyapunov Krasovskii functional structures and efficient results on simulations. Another approach widely used in the literature stems from the robust analysis which use the same driving forces namely the comparisons systems and integral inequalities [18]. In [16], a combined full block $\mathrm{S}$ procedure and Lyapunov analysis is performed to prove the stability of a distributed delay system with a rational kernel. More recently, using quadratic separation approach, [7] has provided some LMI criteria for polynomial kernels. In this paper, we aim at extending the procedure proposed recently in [8] to the case of distributed delay systems and an arbitrary continuous and known kernel. The basic idea is to project this function upon a polynomial set whose a basis is orthogonal like Legendre polynomials. Choosing adequate extra states related to the projection of the kernel upon Legendre polynomials, the original system can be modeled as a classical time delay state submitted to an exogenous bounded perturbation. Using classical procedure in robust analysis, this last system can also be viewed as a linear system connected to a set of operators composed of the operators related to the distributed delayed dynamics. This last part is then embedded into some relevant structured uncertainties whose size is controlled by new inequalities from Bessel inequalities [8].

The paper is organized as follows. Next section is devoted to some preliminaries on the distributed delay system and quadratic separation. Then section 3 formulates a way to describe the distributed delay system as an interconnected uncertain system. Section 4 gives the central results of the paper. Section 5 presents some numerical simulations.

Notations: Throughout the paper, the following notations are used. $1_{\mathrm{n}}$ and $0_{\mathrm{m} \times \mathrm{n}}$ denote respectively the identity matrix of size $n$ and null matrix of size $m \times n$. For two symmetric matrices, $A$ and $B, A>(\geq) B$ means that $A-B$ is (semi-) positive definite. $A^{T}$ denotes the transpose of $A$.

\section{PREliminaries}

\section{A. Definitions}

We define by $\mathcal{H}$ the vector space of complex valued square integrable functions on $[-h, 0]$. For any functions in $\mathcal{H}$, we define the hermitian inner product

$$
\langle f, g\rangle=\int_{-h}^{0} f(\theta) g^{*}(\theta) d \theta
$$


where $f$ and $g$ belong to $\mathcal{H}$. Basically, $x_{i}, x_{j} \in \mathcal{H}, i \neq j$ are said orthogonal if $\left\langle x_{i}, x_{j}\right\rangle=0$. A sequence of $\mathcal{H}$, $\left\{e_{0}, e_{1}, e_{2}, \ldots, e_{n}\right\}$, is an orthonormal sequence if $\left\langle e_{i}, e_{j}\right\rangle=$ $\kappa_{i j}$, where $\kappa_{i j}$ is the kronecker notation. Based on these definitions, we recall the Bessel inequality that will be employed later in the paper.

Lemma 1 (Bessel inequality): Let $\left\{e_{0}, e_{1}, e_{2}, \ldots, e_{n}\right\}$ be an orthonormal sequence in $\mathcal{H}$. Then, for any $f$ in $\mathcal{H}$ the following inequality holds:

$$
\langle f, f\rangle \geq \sum_{i=0}^{n}\left|\left\langle f, e_{i}\right\rangle\right|^{2}
$$

An example of polynomials for the inner product (1) is the well known Legendre polynomials whose definition and properties are stated below.

Definition 1 (Legendre polynomials): The Legendre polynomials considered over the interval $[-h, 0]$ are defined by

$$
\forall k \in \mathbb{N}, \quad L_{k}(u)=(-1)^{k} \sum_{l=0}^{k} p_{l}^{k}\left(\frac{u+h}{h}\right)^{l} .
$$

with $p_{l}^{k}=(-1)^{l}\left(\begin{array}{c}k \\ l\end{array}\right)\left(\begin{array}{c}k+1 \\ l\end{array}\right)$ where $\left(\begin{array}{l}k \\ l\end{array}\right)=\frac{k !}{l !(k-l) !}$. Some properties are summarized below and will be useful in the sequel.

P1

$$
\begin{aligned}
& \text { Orthogonality: } \forall(k, l) \in \mathbb{N}^{2}, \\
& \qquad \int_{-h}^{0} L_{k}(u) L_{l}(u) \mathrm{d} u= \begin{cases}0, & k \neq l \\
\frac{h}{2 k+1}, & k=l\end{cases}
\end{aligned}
$$

P2 Boundary conditions:

$$
\forall k \in \mathbb{N}, \quad L_{k}(0)=1, \quad L_{k}(-h)=(-1)^{k} .
$$

P3 Recurrence on the derivative:

$$
\frac{d}{d u}\left[L_{k+1}(u)-L_{k-1}(u)\right]=\frac{2(2 k+1)}{h} L_{k}(u)
$$

with

$$
\frac{\mathrm{d}}{\mathrm{d} u} L_{0}(u)=0, \quad \frac{\mathrm{d}}{\mathrm{d} u} L_{1}(u)=\frac{2}{h} L_{0}(u) .
$$

Proofs of these properties are standard results and can be found in [6].

\section{B. Problem statement}

Consider the distributed delay system given by:

$$
\dot{x}(t)=A x(t)+A_{d} \int_{-h}^{0} f(\theta) x(t+\theta) d \theta,
$$

where $x \in \mathbb{R}^{n}$ is the instantaneous state vector and $A, A_{d} \in$ $\mathbb{R}^{n \times n}$ are constant matrices. The function $f$ is a scalar continuous function, defined over the interval $[-h, 0]$, and represents the kernel of the distributed delay. We aim at assessing stability of the system (3) using robust analysis, more especially using the quadratic separation approach which has been already used for polynomial kernel in [8] or pointwise delay system in [20].

Remark 1: We have considered that the kernel $f$ is a scalar function but this assumption can be easily extended to the case of matrix kernel by considering several distributed terms with adequate matrices $A_{d}$. This will not be presented in this paper because of space limitation.

\section{Stability analysis via quadratic separation}

As a part of robust control theory, the quadratic separation provides a fruitful framework to address stability of nonlinear and uncertain systems [20], [12]. Recent studies [1] have shown that such a framework allows to propose an original approach to deal with the stability analysis of timedelay systems. The key idea is to reformulate the delay system (3) as an uncertain feedback system as in Figure 1. In our case, the uncertainty stems from some operators related to the delay dynamic. It has been shown that a suitable interconnection modeling, based on relevant choices of operators, may reduce the conservatism of the analysis [1]. The stability conditions presented in this paper are founded on the theorem introduced below.

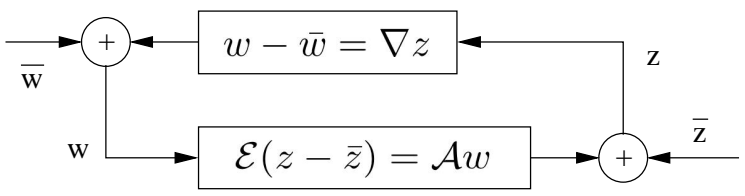

Fig. 1: Feedback system.

Let consider the interconnection defined by Figure 1 where $\mathcal{E}$ and $\mathcal{A}$ are two, real valued, possibly non-square matrices and $\nabla$ is a linear operator which represent the system uncertainty. This latter is assumed to belong to an uncertain set $\mathbb{W}$. In the present paper, we aim at embedding some delay-dependent operators into the matrix $\nabla$. For simplicity of notations, we assume that $\mathcal{E}$ is full column rank. Then, we are interested in looking for conditions that assess the stability of the interconnection.

Theorem 1 ([20]): The uncertain feedback system of Figure 1 is well-posed and stable if and only if there exists a Hermitian matrix $\Theta=\Theta^{*}$ satisfying both conditions

$$
\begin{gathered}
{\left[\begin{array}{cc}
\mathcal{E} & -\mathcal{A}
\end{array}\right]^{\perp *} \Theta\left[\begin{array}{ll}
\mathcal{E} & -\mathcal{A}
\end{array}\right]^{\perp}>0,} \\
{\left[\begin{array}{c}
1 \\
\nabla
\end{array}\right]^{*} \Theta\left[\begin{array}{c}
1 \\
\nabla
\end{array}\right] \leq 0 \quad, \quad \forall \nabla \in \mathbb{W} .}
\end{gathered}
$$

As a result, the stability is evaluated through two matrix inequalities (4) and (5) related respectively to the lower and the upper bloc. Basically, inequality (5) is built from definitions and informations on the system uncertainty. Then, the other one (4) provides the stability condition to be tested.

\section{Modeling THE DELAY SYSTEM AS A FEEDBACK SYSTEM}

\section{A. Projection of the kernel}

In light of the properties of Legendre polynomials, we consider the polynomial approximation of the kernel $f$. For a given integer $r$, it is trivial to write

$$
\forall \theta \in[-h, 0], \quad f(\theta)=\sum_{i=0}^{r} a_{i} L_{i}(\theta)+g_{r}(\theta),
$$


where

$$
\begin{array}{ll}
a_{i} & =\frac{2 i+1}{h} \int_{-h}^{0} f(u) L_{k}(u) \mathrm{d} u, \quad \forall i=0, \ldots, r \\
g_{r}(\theta) & =f(\theta)-\sum_{i=0}^{r} a_{i} L_{i}(\theta) .
\end{array}
$$

Then, the distributed delay system (3) can be reformulated as

$$
\dot{x}(t)=A x(t)+A_{d} \sum_{i=0}^{r} a_{i} x_{i}(t)+A_{d} \int_{-h}^{0} g_{r}(\theta) x(t+\theta) d \theta,
$$

where we define a new state $x_{i}(t)=\int_{-h}^{0} L_{i}(\theta) x(t+\theta) d \theta$.

\section{B. An extended system}

The initial system (3) has been reformulated introducing extra state variables $x_{i}$ whose dynamics are:

$$
\begin{aligned}
\dot{x}_{i}(t)= & \int_{-h}^{0} L_{i}(\theta) \dot{x}(t+\theta) d \theta, \\
= & L_{i}(0) x(t)-L_{i}(-h) x(t-h) \\
& -\int_{-h}^{0} \frac{d}{d \theta} L_{i}(\theta) x(t+\theta) d \theta,
\end{aligned}
$$

as $L_{i-1}(0)=L_{i+1}(0)$ and $L_{i-1}(-h)=L_{i+1}(-h)$, subtracting the $(i-1)$-th to the $(i+1)$-th equation, we have

$$
\begin{aligned}
\dot{x}_{i+1}(t)-\dot{x}_{i-1}(t) & =-\int_{-h}^{0}\left[\dot{L}_{i+1}(\theta)-\dot{L}_{i-1}(\theta)\right] x(t+\theta) d \theta, \\
& =-\frac{2(2 i+1)}{h} x_{i}(t) .
\end{aligned}
$$

The last equality results from property P3 in Definition 1 . So the additional dynamics are expressed as

$$
\dot{x}_{i+1}(t)=\dot{x}_{i-1}(t)-\frac{2(2 i+1)}{h} x_{i}(t),
$$

with the initialization given by:

$$
\begin{aligned}
\dot{x}_{0}(t) & =\int_{-h}^{0} \dot{x}(t+\theta) d \theta=x(t)-x(t-h) \\
\dot{x}_{1}(t) & =\int_{-h}^{0}\left(\frac{2}{h} \theta+1\right) \dot{x}(t+\theta) d \theta \\
& =x(t)+x(t-h)-\frac{2}{h} x_{0}(t) .
\end{aligned}
$$

Let introduce the extra state $\zeta(t)$ and $x_{g}(t)$.

$$
\zeta(t)=\left[\begin{array}{c}
x_{0}(t) \\
x_{1}(t) \\
\vdots \\
x_{r}(t)
\end{array}\right], \quad x_{g}(t)=\int_{-h}^{0} g_{r}(\theta) x(t+\theta) d \theta
$$

The dynamics of the extended distributed delay system can be easily described as follows:

$$
\begin{aligned}
\dot{x}(t) & =A x(t)+\bar{A}_{d r} \zeta(t)+A_{d} x_{g}(t), \\
& \left.\begin{array}{ll|l|l}
1_{2 \mathrm{n}} & 0_{2 \mathrm{n} \times(\mathrm{r}-1) \mathrm{n}} \\
\hline & E
\end{array}\right] \dot{\zeta}(t)=\left[\begin{array}{c|c}
1_{\mathrm{n}} & 0_{\mathrm{n} \times(\mathrm{r}+1) \mathrm{n}} \\
\hline 1_{\mathrm{n}} & U \\
0_{(\mathrm{r}-1) \mathrm{n} \times \mathrm{n}} & U
\end{array}\right] \zeta(t) \\
& +\left[\begin{array}{c}
-1_{\mathrm{n}} \\
1_{\mathrm{n}} \\
0_{(\mathrm{r}-1) \mathrm{n} \times \mathrm{n}}
\end{array}\right] x(t-h),
\end{aligned}
$$

with

$$
\begin{aligned}
\bar{A}_{d r} & =\left[\begin{array}{llll}
a_{0} A_{d} & a_{1} A_{d} & \ldots & a_{r} A_{d}
\end{array}\right], \\
U & =\left[\begin{array}{cccc|c}
\alpha_{0} 1_{\mathrm{n}} & \ldots & 0_{\mathrm{n}} & 0_{\mathrm{n}} \\
& \ddots & \vdots & \\
& & & \alpha_{r-1} 1_{\mathrm{n}} & 0_{\mathrm{n}}
\end{array}\right], \\
E & =\left[\begin{array}{ccccc}
0_{\mathrm{n}} & 0 & 1 & & 0 \\
& \ddots & \ddots & \ddots & \\
0 & & -1 & 0 & 1
\end{array}\right],
\end{aligned}
$$

and $\alpha_{i}=-2 \frac{2 i+1}{h}$. We obtain therefore a time delay system whose stability will be studied via the quadratic separation approach.

\section{Uncertain feedback system}

The methodology consists in expressing the delay system (3) into an interconnected system as depicted in Figure 1. To this end, we need to list all operators that are involved in the dynamic of the system as well as uncertainties. First of all, let define the following operators, for $i=\{0,1,2, \ldots, r\}$

$$
\begin{aligned}
\delta_{i}: \quad \mathbb{R} & \rightarrow \mathbb{R}, \\
x(t) & \rightarrow \int_{-h}^{0} L_{i}(\theta) x(t+\theta) d \theta, \\
\delta_{g}: \quad \mathbb{R} & \rightarrow \mathbb{R}, \\
x(t) & \rightarrow \int_{-h}^{0} g(\theta) x(t+\theta) d \theta .
\end{aligned}
$$

The first operator is the operator that defines state variables $x_{i}, x_{i}(t)=\delta_{i}[x](t)$. The second operator is associated to the remainder of the projection of the kernel $f$, namely $x_{g}(t)=$ $\delta_{g}[x](t)$. Since $h$ is constant, the operators above can be expressed in the Laplace domain as $\delta_{i}(s)=\int_{-h}^{0} L_{i}(\theta) e^{s \theta} d \theta$ and $\delta_{g}(s)=\int_{-h}^{0} g_{r}(\theta) e^{s \theta} d \theta$. Now, we gather all the operators that describe (3) according to our modeling:

$$
\underbrace{\left[\begin{array}{c}
x(t) \\
\zeta(t) \\
x(t-h) \\
\zeta(t) \\
x_{g}(t)
\end{array}\right]}_{w(t)}=\nabla \underbrace{\left[\begin{array}{c}
\dot{x}(t) \\
\dot{\zeta}(t) \\
x(t) \\
x(t) \\
x(t)
\end{array}\right]}_{z(t)},
$$

with $\bar{\delta}=\left[\delta_{0}, \delta_{1}, \ldots, \delta_{r}\right]^{T}$ and

$$
\nabla=\left[\begin{array}{cccc}
s^{-1} 1_{\mathrm{n}(\mathrm{r}+2)} & 0 & 0 & 0 \\
0 & e^{-s h} 1_{\mathrm{n}} & 0 & 0 \\
0 & 0 & \bar{\delta} \otimes 1_{\mathrm{n}} & 0 \\
0 & 0 & 0 & \delta_{g} 1_{\mathrm{n}}
\end{array}\right] .
$$

Combining expressions (8) and (9), we can build the linear equation (feedforward block in Figure 1) specifying all the relationships between vectors $z$ and $w$ :

$$
\mathcal{E}\left[\begin{array}{c}
\dot{x}(t) \\
\dot{\zeta}(t) \\
x(t) \\
x(t) \\
x(t)
\end{array}\right]=\mathcal{A}\left[\begin{array}{c}
x(t) \\
\zeta(t) \\
x(t-h) \\
\zeta(t) \\
x_{g}(t)
\end{array}\right]
$$


with

$$
\mathcal{E}=\left[\begin{array}{ccc}
1_{3 n} & 0_{3 n \times(r-1) n} & 0_{3 n} \\
0_{(r-1) n \times n} & E & 0_{(r-1) n \times 3 n} \\
0_{3 n} & 0_{3 n \times(r-1) n} & 1_{3 n} \\
\hline 0 & 0 & 0
\end{array}\right],
$$

\begin{tabular}{|c|c|c|c|c|}
\hline \multicolumn{5}{|c|}{$\mathcal{A}=$} \\
\hline$A$ & $\bar{A}_{d r}$ & $0_{n}$ & $0_{n \times(r+1) n}$ & $A_{d}$ \\
\hline $1_{n}$ & $0_{n \times(r+1) n}$ & $-1_{n}$ & $0_{n \times(r+1) n}$ & $0_{n}$ \\
\hline $\begin{array}{c}1_{n} \\
0_{(r-1) n \times n}\end{array}$ & 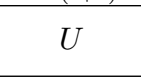 & $\begin{array}{c}1_{n} \\
0_{(r-1) n \times n}\end{array}$ & $0_{r n \times(r+1) n}$ & $0_{r n \times n}$ \\
\hline $\begin{array}{l}1_{\mathrm{n}} \\
1_{\mathrm{n}} \\
1_{\mathrm{n}}\end{array}$ & $0_{3 n \times(r+1) n}$ & $0_{3 n \times n}$ & $0_{3 n \times(r+1) n}$ & $0_{3 n \times n}$ \\
\hline $0_{n(r+1)}$ & $1_{n(r+1)}$ & $0_{n(r+1) \times n}$ & $-1_{n(r+1)}$ & $0_{n(r+1) \times}$ \\
\hline
\end{tabular}

IV. STABILITY CONDITION

\section{A. Inequality constraints}

The dynamic of our feedback system is described by a set of operators (11). We want to state now a set of inequalities that will be useful to build the constraint on $\nabla$ (5).

Lemma 2 ([1]): A quadratic constraint for the operator $s^{-1}$ is given by the following inequality for any positive definite matrix $P$,

$$
\left[\begin{array}{c}
1_{\mathrm{n}} \\
s^{-1} 1_{\mathrm{n}}
\end{array}\right]^{*}\left[\begin{array}{cc}
0 & -P \\
-P & 0
\end{array}\right]\left[\begin{array}{c}
1_{\mathrm{n}} \\
s^{-1} 1_{\mathrm{n}}
\end{array}\right] \leq 0 .
$$

Lemma 3 ([1]): A quadratic constraint for the operator $e^{-h s}$ is given by the following inequality for any positive definite matrix $Q$,

$$
\left[\begin{array}{c}
1_{\mathrm{n}} \\
e^{-h s} 1_{\mathrm{n}}
\end{array}\right]^{*}\left[\begin{array}{cc}
-Q & 0 \\
0 & Q
\end{array}\right]\left[\begin{array}{c}
1_{\mathrm{n}} \\
e^{-h s} 1_{\mathrm{n}}
\end{array}\right] \leq 0 .
$$

The proof of the two above lemmas can be found in [20], [1].

Lemma 4: [8] A quadratic constraint for the operator $\bar{\delta}$ is given by the following inequality for any positive definite matrix $R_{1}$,

$$
\left[\begin{array}{c}
1_{\mathrm{n}} \\
\delta_{0} 1_{\mathrm{n}} \\
\delta_{1} 1_{\mathrm{n}} \\
\vdots \\
\delta_{r} 1_{\mathrm{n}}
\end{array}\right]^{*} \Xi\left[\begin{array}{c}
1_{\mathrm{n}} \\
\delta_{0} 1_{\mathrm{n}} \\
\delta_{1} 1_{\mathrm{n}} \\
\vdots \\
\delta_{r} 1_{\mathrm{n}}
\end{array}\right] \leq 0
$$

with

$$
\Xi=\left[\begin{array}{ccccc}
-h^{2} R_{1} & 0 & 0 & \ldots & 0 \\
0 & R_{1} & 0 & \cdots & 0 \\
0 & 0 & 3 R_{1} & & 0 \\
\vdots & \vdots & & \ddots & \\
0 & 0 & 0 & & (2 r+1) R_{1}
\end{array}\right]
$$

Proof: Let apply the Bessel inequality (Lemma 1) on the delay function $e^{s \theta}$ with the normalized Legendre polynomials $\sqrt{\frac{2 i+1}{h}} L_{i}$ :

$$
\sum_{i=0}^{r}\left|\left\langle e^{s \theta}, \sqrt{\frac{2 i+1}{h}} L_{i}\right\rangle\right|^{2} \leq\left\langle e^{s \theta}, e^{s \theta}\right\rangle
$$

Upperbounding $\left\langle e^{s \theta}, e^{s \theta}\right\rangle$ by $h$, we obtain:

$$
\sum_{i=0}^{r}(2 i+1) \delta_{i} \delta_{i}^{*} \leq h\left\langle e^{s \theta}, e^{s \theta}\right\rangle \leq h^{2},
$$

The proof follows straightforwardly.

Lemma 5: A quadratic constraint for the operator $\delta_{g}$ is given by the following inequality for any positive definite matrix $R_{2}$,

$$
\left[\begin{array}{c}
1_{\mathrm{n}} \\
\delta_{g} 1_{\mathrm{n}}
\end{array}\right]^{*}\left[\begin{array}{cc}
-\lambda R_{2} & 0 \\
0 & R_{2}
\end{array}\right]\left[\begin{array}{c}
1_{\mathrm{n}} \\
\delta_{g} 1_{\mathrm{n}}
\end{array}\right] \leq 0
$$

with $\lambda=h \int_{-h}^{0} \mid g_{r}\left(\left.\theta\right|^{2} d \theta\right.$.

Proof: Let apply the Cauchy-Schwartz inequality on the function $\delta_{g}$ :

$$
\delta_{g} \delta_{g}^{*}=\left|\int_{-h}^{0} g_{r}(\theta) e^{s \theta} d \theta\right|^{2} \leq \lambda,
$$

which concludes the proof.

\section{B. Main result}

We now propose a theorem that provides a sufficient stability condition for the distributed delay system system (3). This stability condition is expressed as a LMI condition.

Theorem 2: For a given positive scalar $h$ and a positive integer $r$, if there exist positive definite matrices $P \in$ $\mathbb{R}^{n(r+2) \times n(r+2)}, Q, R_{1}$ and $R_{2} \in \mathbb{R}^{n \times n}$, then the system (3) is asymptotically stable if the following LMI is satisfied:

$$
\left[\begin{array}{ll}
\mathcal{E} & -\mathcal{A}
\end{array}\right]^{\perp *} \Theta\left[\begin{array}{ll}
\mathcal{E} & -\mathcal{A}
\end{array}\right]^{\perp}>0,
$$

where matrices $\mathcal{E}, \mathcal{A}$ and $\Theta$ are defined in (12) and (13).

Proof: In Section III-C, it has been shown that system (3) could be expressed as in Figure 1 with equations (11)-(12). Combining all the quadratic constraints stated in Section IV-A, we build the matrix (the separator)

$$
\Theta=\left[\begin{array}{ll}
\Theta_{11} & \Theta_{12} \\
\Theta_{12}^{T} & \Theta_{22}
\end{array}\right]
$$

with

$$
\Theta_{11}=\left[\begin{array}{ccccc}
0 & 0 & 0 & 0 \\
0 & -Q & 0 & 0 \\
0 & 0 & -h^{2} R_{1} & 0 \\
0 & 0 & 0 & -\lambda R_{2}
\end{array}\right],
$$




\begin{tabular}{|c|c|c|}
\hline Theorems & $h$ & nbr. of var. \\
\hline \hline [3] ("analytical") & 1.498 & - \\
\hline$[27]$ & 1.03 & 3 \\
\hline Th.2 $(r=1)$ & 1.39 & 9 \\
\hline Th.2 $(r=2)$ & 1.491 & 13 \\
\hline Th.2 $(r=3)$ & 1.495 & 18 \\
\hline
\end{tabular}

TABLE I: Maximal allowable delay $h$ for system (14).

that fulfills condition (5). Hence, invoking Theorem 1, the aforementioned system is stable if the first condition (4) is also satisfied.

Remark 2: Following [9] or [18], we can show that the proposed result can also be interpreted as a LyapunovKrasovskii functional of the form:

$$
\begin{aligned}
V= & {\left[\begin{array}{l}
x(t) \\
\zeta(t)
\end{array}\right]^{T} P\left[\begin{array}{l}
x(t) \\
\zeta(t)
\end{array}\right]+\int_{-h}^{0} x_{t}^{T}(\theta) Q x_{t}(\theta) d \theta } \\
& +\int_{-h}^{0} \int_{s}^{0} x_{t}^{T}(\theta) R_{1} x_{t}(\theta) d \theta d s \\
& +\int_{-h}^{0} \int_{s}^{0} x_{g} T(t+\theta) R_{2} x_{g}(t+\theta) d \theta d s
\end{aligned}
$$

It appears that this functional is related to the functional used recently by [22] and [23], [24] to establish some stability criteria using either Wirtinger inequalities or Bessel inequalities.

\section{NUMERICAL EXAMPLES}

\section{A. Example 1}

Let consider a first scalar example:

$$
\dot{x}(t)=-x(t)+\int_{-h}^{0} e^{-\theta} \sin (\theta) x(t+\theta) d \theta
$$

Using a numerical method [2], [3], system (14) is asymptotically stable for all delays less than 1.498. Table I shows results obtained with Theorem 2. As expected, better results are obtained as the degree of the polynomial $r$ increases. Figure 2 shows the kernel function $f(\theta)$ and the projection polynomial $p_{r}(\theta)=\sum_{0}^{r} a_{i} L_{i}(\theta)$. The difference between the two curves represents $g_{r}(\theta)$ and it can be seen that for $r=2$, the approximation is good enough to get a small $\lambda$.

\section{B. Example 2}

Let consider the following distributed delay system:

$$
\dot{x}(t)=-2 x(t)+\int_{-h}^{0}(\theta+3 \cos \theta) x(t+\theta) d \theta
$$

This example is interesting because it has two stability pockets namely $h$ in $[0,0.964]$ and in [1.372, 2.105] [3]. Table II shows results obtained with Theorem 2. Note that even if the theorem from [27] provides a very good result in estimating the first interval of stability, it is not able to detect the second one.

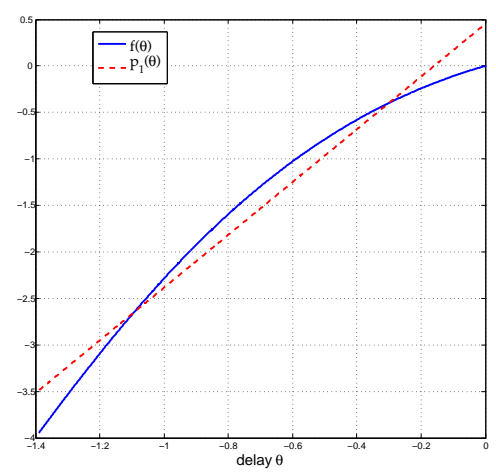

(a)

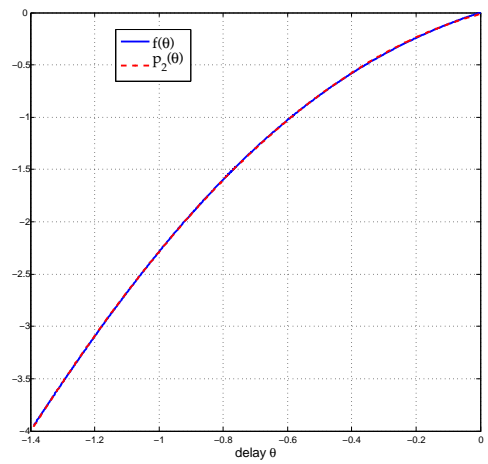

(b)

Fig. 2: The delay kernel $f$ and the projection polynomial $p_{r}$ (for $r=1$ and $r=2$ ).

\begin{tabular}{|c|c|c|c|}
\hline Theorems & $1^{\text {st }}$ interval & $2^{\text {nd }}$ interval & nbr of var. \\
\hline \hline$[3]$ ("analytical") & {$[0-0.964]$} & {$[1.372-2.105]$} & - \\
\hline$[27]$ & {$[0-0.964]$} & - & 3 \\
\hline Th.2 $(r=1)$ & {$[0-0.86]$} & - & 9 \\
\hline Th.2 $(r=2)$ & {$[0-0.960]$} & {$[1.42-1.99]$} & 13 \\
\hline Th.2 $(r=3)$ & {$[0-0.964]$} & {$[1.375-2.097]$} & 18 \\
\hline
\end{tabular}

TABLE II: Allowable delay $h$ for system (15).

\section{Example 3}

The system we are considering now is interesting in that it is unstable if the delay $h$ is zero.

$$
\dot{x}(t)=0.2 x(t)-\int_{-h}^{0}(1-0.3 \theta) x(t+\theta) d \theta
$$

In this case, although the dynamic matrix $A$ (here a scalar) is positive, the delayed dynamic may stabilize the system. [27] shows that system (16) is stable for $h$ in $[0.195-1.442]$. Invoking Theorem 2 with $r=1$, a larger stability interval: $[0.195-1.658]$ is obtained. Hence, it is worthy to note that both results are able to assess the stability of the distributed delay system even when the delay-free system is unstable.

\section{Example 4}

This last example is extracted from [17]:

$$
\dot{x}(t)=-2.1 x(t)-\int_{-h}^{0} \tilde{f}(\theta) x(t+\theta) d \theta,
$$


with an uncertain kernel $\tilde{f}(\theta)=f(\theta)+\Delta_{f}(\theta)$ and $f(\theta)=$ $\frac{-0.6 \theta}{1+\theta^{2}}$. It is assumed that $\left\|\Delta_{f}(\theta)\right\| \leq 0.1$ for all $\theta \in[-h, 0]$. In order to handle this uncertainty and apply our result, a slight change on operator $\delta_{g}$ is required:

$$
\delta_{g}(s)=\int_{-h}^{0}\left(g(\theta)+\Delta_{f}(\theta)\right) e^{s \theta} d \theta
$$

and we have the inequality

$$
\delta_{g}^{*} \delta_{g} \leq h \int_{-h}^{0}|g(\theta)|^{2}+\left|\Delta_{f}(\theta)\right|^{2} d \theta
$$

Hence, Theorem 2 can be applied, adapting the scalar $\lambda$ in (13). The stability condition in [17] states that (17) is stable for a delay interval $h$ up to 5.2. The result in this paper is able to find out a larger delay interval (for which the system remains stable): $h=7.8$ for $r=2$ and $h=8$ for $r=4$.

\section{CONCLUSION}

In this paper, we have provided a systematic procedure for the stability analysis of distributed delay systems with a general kernel using on a robust approach. The approach is based on the approximation of the kernel by orthogonal polynomials. The resulting system is then rewritten as a more classical time delay system submitted to a perturbation whose size can be estimated by integral inequalities issued from Bessel inequalities. The resulting criterion is expressed in terms of an LMI and give interesting results in simulations. Future works include the conservatism reduction analysis and the more tricky case of time-varying delay.

\section{REFERENCES}

[1] Y. Ariba, F. Gouaisbaut, and K.H. Johansson. Robust stability of time-varying delay systems: The quadratic separation approach. Asian Journal of Control, 14(5):1205-1214, 2012.

[2] D. Breda, S. Maset, and R. Vermiglio. Pseudospectral differencing methods for characteristic roots of delay differential equations. SIAM Journal on Scientific Computing, 27(2):482-495, 2005.

[3] D. Breda, S. Maset, and R. Vermiglio. Trace-dde: a tool for robust analysis and characteristic equations for delay differential equations. In Topics in Time Delay Systems, volume 388 of Lecture Notes in Control and Information Sciences, pages 145-155. Springer Berlin Heidelberg, 2009.

[4] W.-H. Chen and W.X. Zheng. Delay-dependent robust stabilization for uncertain neutral systems with distributed delays. Automatica, 43(1):95 - 104, 2007.

[5] E. Fridman and U Shaked. A descriptor system approach to $\mathcal{H}_{\infty}$ control of linear time-delay systems. IEEE Trans. on Automatic Control, 47:253-270, February 2002.

[6] W. Gautschi. Orthogonal Polynomials, Computation and Approximation. Oxford Science, 2004.

[7] F Gouaisbaut and Y Ariba. Delay range stability of a class of distributed time delay systems. Systems \& Control Letters, 60(3):211$217,2011$.

[8] F. Gouaisbaut, Y. Ariba, and A. Seuret. Bessel inequality for robust stability analysis of time-delay system. In Decision and Control (CDC), 2013 IEEE 52nd Annual Conference on, pages 928-933, Dec 2013.

[9] F. Gouaisbaut and D. Peaucelle. Delay-dependent robust stability of time delay systems. In $5^{\text {th }}$ IFAC Symposium on Robust Control Design (ROCOND'06), Toulouse, France, July 2006.

[10] K. Gu, Q.L. Han, A.C.J. Luo, and S.I. Niculescu. Discretized lyapunov functional for systems with distributed delay and piecewise constant coefficients. International Journal of Control, 74(7):737 - 744, 2001.
[11] Q.-L. Han. A descriptor system approach to robust stability of uncertain neutral systems with discrete and distributed delays. Automatica, 40(10): 1791 - 1796, 2004.

[12] T. Iwasaki and S. Hara. Well-posedness of feedback systems: insights into exact robustnessanalysis and approximate computations. IEEE Trans. on Automatic Control, 43:619-630, May 1998.

[13] V. B. Kolmanovskii and L. E. Shaikhet. Control of systems with aftereffect. American Mathematical Society, 1996.

[14] X.-G. Li and X.-J. Zhu. Stability analysis of neutral systems with distributed delays. Automatica, 44(8):2197 - 2201, 2008.

[15] C.-I. Morarescu, S.-I. Niculescu, and Gu K. Stability crossing curves of shifted gamma-distributed delay systems. SIAM Journal on Applied Dynamical Systems, 6(2):475-793, 2007.

[16] U. Münz, J. Rieber, and F. Allgöwer. Topics in Time-Delay Systems: Analysis, Algorithms, and Control, volume 388 of LNCIS, chapter Robust Stabilization and $\mathcal{H}_{\infty}$ Control of Uncertain Distributed Delay Systems, pages 221-231. Springer, Berlin, 2009.

[17] U. Münz, J. M. Rieber, and F. Allgöwer. Robust stability of distributed delay systems. In Proc. 17th IFAC World Congress, pages 1235412358, Seoul, Korea, July 2008.

[18] S.I. Niculescu. Delay Effects on Stability. A Robust Control Approach, volume 269 of Lecture Notes in Control and Information Sciences. Springer-Verlag, Heildelberg, 2001.

[19] H. Ozbay, C. Bonnet, and J. Clairambault. Stability analysis of systems with distributed delays and application to hematopoietic cell maturation dynamics. In Decision and Control, 2008. CDC 2008. 47th IEEE Conference on, pages 2050-2055, Dec 2008.

[20] D Peaucelle, D Arzelier, D Henrion, and F Gouaisbaut. Quadratic separation for feedback connection of an uncertain matrix and an implicit linear transformation. Automatica, 43(5):795-804, 2007.

[21] O. Santos, S. Mondie, and V.L. Kharitonov. Robust stability conditions for systems with distributed delays. In Decision and Control, 2006 45th IEEE Conference on, pages 217-222, Dec 2006.

[22] A. Seuret and F. Gouaisbaut. Wirtinger-based integral inequality: Application to time-delay systems. Automatica, 49(9):2860-2866, 2013.

[23] A. Seuret and F. Gouaisbaut. Complete quadratic lyapunov functionals using bessel-legendre inequality. In Control Conference (ECC), 2014 European, pages 448-453, June 2014.

[24] A. Seuret and F. Gouaisbaut. Hierarchy of LMI conditions for the stability analysis of time delay systems. Technical Report LAAS Report 14429, Jun 2014. This article has been submitted to Systems \& Control Letters.

[25] R Sipahi, F Atay, and S Niculescu. Stability of Traffic Flow Behavior with Distributed Delays Modeling the Memory Effects of the Drivers. SIAM Journal on Applied Mathematics, 68(3):738-759, 2008.

[26] R. Sipahi, S. Niculescu, C.T. Abdallah, W. Michiels, and Keqin Gu. Stability and stabilization of systems with time delay. Control Systems, IEEE, 31(1):38 -65, feb. 2011.

[27] O. Solomon and E. Fridman. New stability conditions for systems with distributed delays. Automatica, 49(11):3467 - 3475, 2013.

[28] L. Xie, E. Fridman, and U. Shaked. A robust $\mathcal{H}_{\infty}$ control of distributed delay systems with application to combustion control. IEEE Trans. on Automatic Control, 46(12):1930-1935, 2001. 